\title{
Optimal Numerical Realization of the Energy Balance Equation for Wind Wave Models
}

\author{
Igor V. Lavrenov \\ State Research Centre of the Russian Federation \\ Arctic and Antarctic Research Institute \\ lavreneaari.nw.ru
}

\begin{abstract}
The optimal numerical realization of the energy balance equation in wind wave models is proposed. The scheme is separated into two parts: the numerical source term integration and the energy propagation numerical realization. The first one is based on a spreading numerical method. Semianalytical solution is used for integration of source term, which includes the wind wave input, dissipation term and exact non-linear energy transfer function. The energy propagation numerical realization is based on the utilisation of the diffusive operator, which is implemented in the semi-Lagrangian numerical method. It gives opportunity to remove the garden-sprinkler effect of the energy balance equation solution for the case of wind wave propagation in the ocean. The new technique yields accurate results and has the additional advantage of being numerically stable, thereby allowing the use of a large time step (three hours and more). The method could be regarded as a general alternative for the numerical realization of energy balance equation in the models.
\end{abstract}

\section{Introduction}

The successful solution of the hindcast or forecast wind wave problem depends on the quality of physical model and on the equation numerical realization in the mathematical modelling. The errors of the solution depend on the accuracy of wind speed calculation as well. Nevertheless the errors of the energy balance equation numerical realization can be no less than the errors coming from the wind value accuracy and from the unsatisfactory implementation of the wind wave physics in the model $[1,2,3,4,6,7]$. In the present study the optimal numerical realization of the energy balance equation in wind wave models is proposed, which gives an opportunity to get reliable results by using sufficient large time step of numerical integration.

\section{Formulation of the Problem}

The evolution of a two-dimensional ocean wave spectrum $S(\omega, \beta, \varphi, \theta, t)$ with respect to frequency $\omega$ and direction $\beta$ (measured here counter clockwise from the parallel) as a function of latitude $\varphi$ and longitude $\theta$ is described by the transport equation $[3,4]$ : 


$$
\frac{\partial S}{\partial t}+\frac{1}{\cos \varphi} \frac{\partial(\varphi \cos \varphi S)}{\partial \varphi}+\frac{\partial(\theta S)}{\partial \theta}+\frac{\partial(\beta S)}{\partial \beta}=G,
$$

where $G=G(\omega, \beta, \varphi, \theta, t)$ is the source function, describing wind input, dissipation and non-linear wave-wave interaction. The functions $\dot{\varphi}, \dot{\theta}, \dot{\beta}$ represent the rates of change of location $(\varphi, \theta)$ and direction of propagation $\beta$ of a wave packet travelling along a ray on spherical surface.

$$
\varphi=C_{g} \sin \beta / R, \dot{\theta}=C_{g} \cos \beta / R \cos \varphi, \beta=-C_{g} \tan \varphi \cos \beta / R
$$

where $C_{g}$ is a group velocity and $R$ is an Earth radius.

The scheme of numerical realization of the energy balance equation (1) with (2) is separated into two parts: the energy propagation numerical realization and the numerical source term integration.

\section{Numerical Scheme of the Wave Energy Propagation}

The numerical solution of the energy balance equation (1) requires the discretization of the continuous wave spectrum $S(\omega, \beta)$ in the frequency-direction space. The mean energy contained in the frequency $\left(\omega_{k}-0.5 \Delta \omega \leq \omega \leq \omega_{k}+0.5 \omega\right)$ and in the direction bands $\left(\beta_{l}-0.5 \Delta \beta \leq \beta \leq \beta_{l}+0.5 \Delta \beta\right)$ can be determined as:

$$
\bar{S}\left(\omega_{k}, \beta_{l}\right)=\frac{1}{\Delta \beta \Delta \omega} \int_{\omega_{k}-\frac{\Delta \omega}{2} \beta_{l}-\frac{\Delta \beta}{2}}^{\omega_{k}+\frac{\Delta \omega}{2} \beta_{l}+\frac{\Delta \beta}{2}} \int^{2} S(\omega, \beta) d \quad \beta d \omega .
$$

In order to derive the mean energy approximation in the bands the bi-quadratic interpolation is applied between points $\omega_{k-1}, \omega_{k}, \omega_{k+1}, \beta_{l-1}, \beta_{l}$ and $\beta_{l+1}$.

$$
\bar{S}\left(\omega_{k}, \beta_{l}\right)=\sum_{i, j=-1}^{1} a_{i} b_{j} S\left(\omega_{k+i}, \beta_{l+j}\right),
$$

where $a_{1}=a_{-1}=b_{-1}=b_{1}=1 / 24 ; a_{0}=b_{0}=1112$. In a similar manner the energy balance equation (1) can be adapted to incorporate the effects of a finite frequencyangular resolution: 


$$
\begin{aligned}
& \frac{\partial S}{\partial t}+\frac{\partial S}{\partial \varphi}(1+\varepsilon 2+\delta) \varphi+\frac{\partial S}{\partial \theta}(1+\varepsilon 2+\delta) \theta+\frac{\partial S}{\partial \beta}(1-\varepsilon 2+\delta) \beta+ \\
& +\varepsilon \frac{C_{g}}{R}\left[\frac{\partial}{\partial \beta}\left\{\cos \beta \frac{\partial S}{\partial \varphi}-\frac{\sin \beta}{\cos \varphi} \frac{\partial \bar{S}}{\partial \theta}\right\}+\tan \varphi \sin \beta \frac{\partial^{2} \bar{S}}{\partial \beta^{2}}\right]- \\
& -\delta \frac{C_{g} \omega}{R} \frac{\partial}{\partial \omega}\left[\left\{\sin \beta \frac{\partial \bar{S}}{\partial \varphi}+\frac{\cos \beta}{\cos \varphi} \frac{\partial \bar{S}}{\partial \theta}\right\}-\tan \varphi \frac{\partial}{\partial \beta}\{\cos \beta \bar{S}\}\right]+ \\
& +O\left(\varepsilon^{2}\right)+O\left(\delta^{2}\right)=G(\bar{S}),
\end{aligned}
$$

where $\varepsilon=(\Delta \beta)^{2} / 12<<1, \delta=(\Delta \omega \omega)^{2} / 12<<1$. It is supposed that $\varepsilon \sim \delta$. Unlike to (1) the equation (5) contains correction terms of first order of small parameters $\mathcal{E}$ and $\delta$, which allow taking into account the fine value of the angular and frequency discretization of spectrum.

Using (2) and (5) one can derive the energy balance equation, which includes the main correction term in the form:

$$
\frac{\partial \bar{S}}{\partial t}+\frac{\partial \bar{S}}{\partial \varphi} \dot{\varphi}+\frac{\partial \bar{S}}{\partial \theta} \dot{\tilde{\theta}}+\frac{\partial \bar{S}}{\partial \beta} \dot{\beta}-A \frac{\varepsilon C_{g}}{R} \frac{\partial^{2} \bar{S}}{\partial \beta^{2}}=G(\bar{S}),
$$

where

$$
A=R / L-\tan \varphi \sin \beta,
$$

$$
\dot{\varphi}=\varphi(1+\varepsilon / 2)
$$

$$
\dot{\tilde{\theta}}=\theta(1+\varepsilon / 2)
$$

$\dot{\beta}=\beta(1-\varepsilon / 2)$,

$L$ is scale of wave propagation. The term correcting the left side of the energy transfer equation (6) is approximated by a simple angular diffusive operator, which describes a slight "exchange" of the energy between the nearest angular components.

The numerical realization of the energy propagation part of the equation (1) results in numerical solution of the equation (5) or (6). The numerical scheme is based on semiLagrangian method in combination with utilisation of angular diffusive operator (INTERPOL method [4,5]).

Let us consider the swell propagation to the South from the initial disturbance, which centre is located at point $\theta_{0}=0^{0}, \varphi_{0}=72^{\circ}$. The wave height partial distribution is approximated by the function $\exp \left(-\delta r / L_{\max }\right)$, where $\delta r$ is the distance from centre, $L_{\max }$ is a correlation radius. The significant wave height at the centre is $10 \mathrm{~m}$, mean wave period is $15 \mathrm{~s}$. The propagation problem is solved by different methods: analytically, using the first order upwind scheme (FOUS), which is used in the WAM model [3], and by the INTERPOL method as well.

The initial partial distribution of significant wave height is shown in fig.1a. The wave height distributions after $24 \mathrm{~h}$ of propagation obtained by the analytical solution, the FOUS and the INTERPOL method (the latter two both using 12 directions and a time step of $30 \mathrm{~min}$ ) are presented in fig.1b, 1c and 1d, respectively). In the FOUS and, to a lesser extent, the INTERPOL results, most of the wave energy tends to concentrate 
along the model direction nearest to the initial direction of propagation. In this direction wave heights are overestimated, while they are underpredicted in other directions. This is "garden-sprinkler" effect [1,4,5] caused by the crude angular resolution of the model. For FOUS solution with 12 directions the local errors can rise to $40 \%$ in the direction of wave propagation, and to $-35 \%$ in other directions. During time the errors along the model direction nearest to the path of propagation grow to $+25 \%$ within $12 \mathrm{~h}$, and at certain points exceed the $+90 \%$ level at $\mathrm{t}=48 \mathrm{~h}$. The negative error levels involve in a less spectacular manner, from peak values of 15$20 \%$ at $\mathrm{t}=12$ to $-40 \%$ at $\mathrm{t}=48 \mathrm{~h}$ of propagation. Increasing the number of directions in the FOUS to 24 reduces the error levels by roughly half. The INTERPOL method with 12 directions is in better agreement with the analytical solution than its FOUS counterpart; its accuracy is comparable to that of the FOUS with 24 directions.
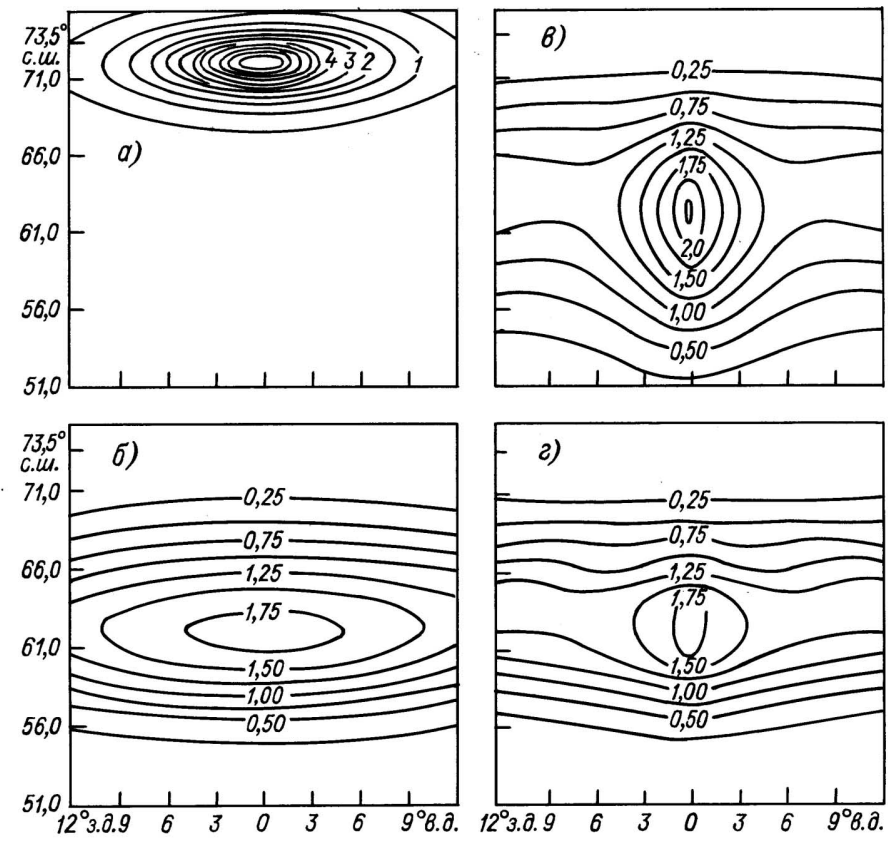

Fig. 1. The spatial distribution of wave heights: $1 \mathrm{a}$ - initial distribution at $t=0 \mathrm{~h} ; 1 \mathrm{~b}$ - analytical solution at $t=24 \mathrm{~h}, 1 \mathrm{c}$ - solution by FOUS with 12 direction at $t=24 \mathrm{~h}, 1 \mathrm{~d}-$ INTERPOL solution (with 12 directions and a time step of 30 minutes) at $t=24 \mathrm{~h}$.

The INTERPOL method with 24 directions also reduces the error levels by half. Fig.2 shows the temporal evolution of the total wave energy $\sum(t)$, its mean location $\langle\varphi(t)\rangle$, the spatial spreading function $\theta(t)$ and root mean square error RMSE. Both the FOUS and INTERPOL schemes are able to reproduce the behaviour of the total energy and mean position of the wave disturbance fairly well (fig. $2 a, 2 b$ ). At the beginning stages of wave propagation all methods give a growing up RMSE level (fig.2d). The error achieves $8 \%$ level at $12 \mathrm{~h}$ and $20 \%$ at $40 \mathrm{~h}$ for FOUS with 12 
directions. The double increasing of the number of directions diminishes errors by halves for the final stage of wave propagation, but for the beginning stage the error level remains approximately the same. The INTERPOL method with 12 directions and the same time step of 20 minutes gives 5\% error at $t=12.5$ hours and $12 \%$ at 40 hours. The increasing of the time step up to $3 \mathrm{~h}$ gives $3 \%$ RMSE at $\mathrm{t}=12 \mathrm{~h}$ and $11 \%$ error at $40 \mathrm{~h}$ and $10 \%$ for 6 - $\mathrm{h}$ time step. So, the increasing of the time step diminishes the error level.
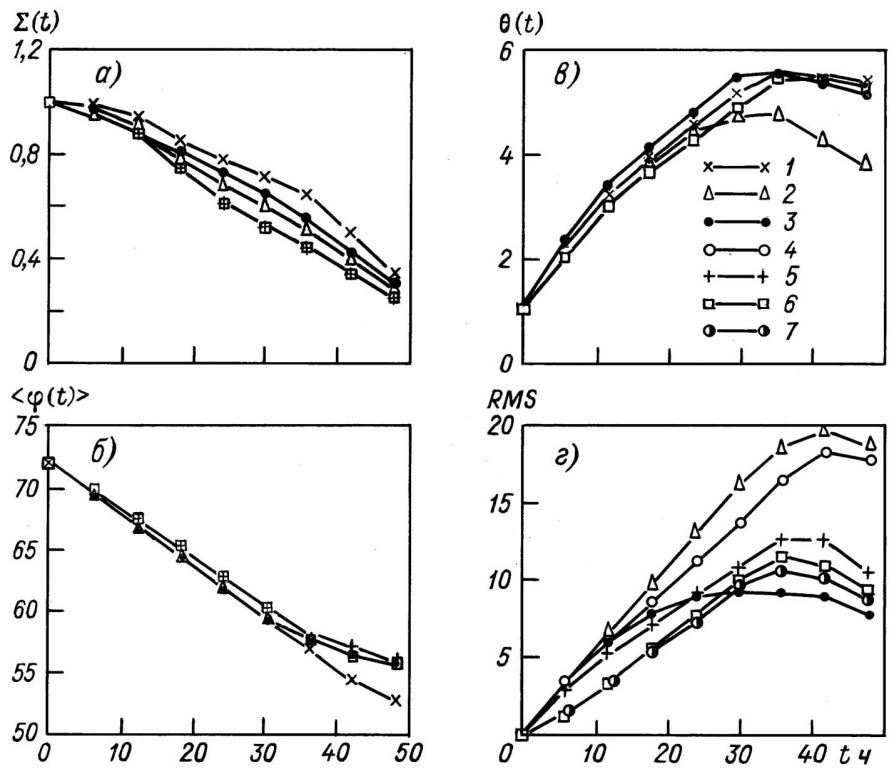

Fig. 2. The evolution of the numerical integral parameters in time. $2 \mathrm{a}$ - the normalized total energy $\Sigma(t) ; 2 \mathrm{~b}$ - the latitude of the mean energy location $\langle\varphi(t)\rangle$, 2c - the spatial spreading function $\theta(t) ; 2 \mathrm{~d}$ - the root mean square error $R M S E(\mathrm{t}) .1$ - analytical solution; 2 - FOUS solution by 12 directions, $\Delta \mathrm{t}=20 \mathrm{~min}$., 3 - FOUS solution by 24 directions, $\Delta \mathrm{t}=$ 20 min.; 4 - FOUS solution by 12 directions, $\Delta \mathrm{t}=20 \mathrm{~min}$. with the angle shift at $\Delta \beta / 2$. 5INTERPOL solution by 12 directions, $\Delta t=20 \mathrm{~min}$. 6 - INTERPOL solution by 12 directions, $\Delta t=3 \mathrm{~h}, 7$ - INTERPOL solution by 12 directions, $\Delta t=6 \mathrm{~h}$.

\section{Numerical Realization of Source Term Numerical Integration}

The second step in total numerical scheme is the numerical realization of the source term integration. The scheme for the next source term is developed:

$$
G=G_{i n}+G_{n l}+G_{d s},
$$


where $G_{i n}$ is the function, describing a wind input, it depends linearly on spectrum $G_{i n}=A S$ [3], where $A$ is the function of frequency $\omega$, angle of spectral component propagation $\beta$ and wind speed $\vec{U} . G_{n l}$ is the exact form of the nonlinear energy transfer computed by optimal algorithms based on the numerical method of integration of the highest precision [4]; $G_{d s}$ is the function of energy dissipation, it consists of two components $G_{d s}=G_{d s}^{1}+G_{d s}^{2}$. The first $G_{d s}^{1}$ is a dissipation mechanism due to white capping, it is the non-linear function of the wave spectrum, it depends on wind speed as well: $G_{d s}^{1}=-A S^{\gamma+1}$ (where $\gamma=\gamma(\omega)$ ). The second component of dissipation is the linear function of the wave spectrum. It doesn't depend on the wind: $G_{d s}^{2}=-B S, B$ is the function of frequency $\omega$, angle of spectral component propagation $\beta$. It can depend on slowly varying integral parameters of the wave spectrum [3].

For the numerical integrating of the source term a spreading algorithm is used. It consists of two steps. At first the simple first order Euler's scheme (or it can be semiimplicit numerical scheme [3] as well) is applied for the numerical integration of equation

$$
\frac{S^{n+\rho}-S^{n}}{\Delta t}=G_{n l}+G_{d s}^{2}
$$

where $0 \leq \rho \leq 1$

At the second step the next finite difference equation is solved

$$
\frac{S^{n+1}-S^{n+\rho}}{\Delta t}=G_{i n}+G_{d s}^{1}
$$

The solution of the equation (9) could be approximated analytically as

$$
S^{n+1}=S^{n+\rho}\left(A\left(S^{n+\rho}\right)^{\gamma} \pm\left|1-A\left(S^{n+\rho}\right)^{\gamma}\right| \mathrm{e}^{-\gamma B \Delta t}\right)^{-1 / \gamma} .
$$

The numerical experiments show that numerical scheme (8)-(10) is able to produce stable numerical results by using time step 1, 3, and 6 hours instead of time step in 20 minutes used in the WAM model. That is why in the present numerical scheme there is no need to introduce any restriction on the wave spectrum and source function numerical values. Such restriction used to include in traditional numerical scheme, because of instability of numerical results [2,3].

Fig. 3 presents the test results of numerical simulations for the frequency spectrum at $t=30 \mathrm{~h}$ duration. The same computations were carried out by the second-order numerical scheme (Adam's method) for the different time step of numerical integration. The comparison results show that results of the second-order numerical scheme for time step in 3 min practically coincide with those of spreading method 
(8)-(10) for time step in $1 \mathrm{~h}$. By increasing the time step up to 3 and 6 hours the numerical value of frequency spectrum remains stable. It is less only by $10 \%$ for time step in $3 \mathrm{~h}$ and by $17 \%$ for time step in $6 \mathrm{~h}$ than appropriate value for time step in $1 \mathrm{~h}$. Fig.4 shows the results of changing the total energy during time for the same methods. The comparison results present the high accuracy of the method (8)-(10) for the time step in $1 \mathrm{~h}$ and $3 \mathrm{~h}$. For large time step the total increasing of energy becomes smaller because of underestimation of non-linear energy transfer contribution.

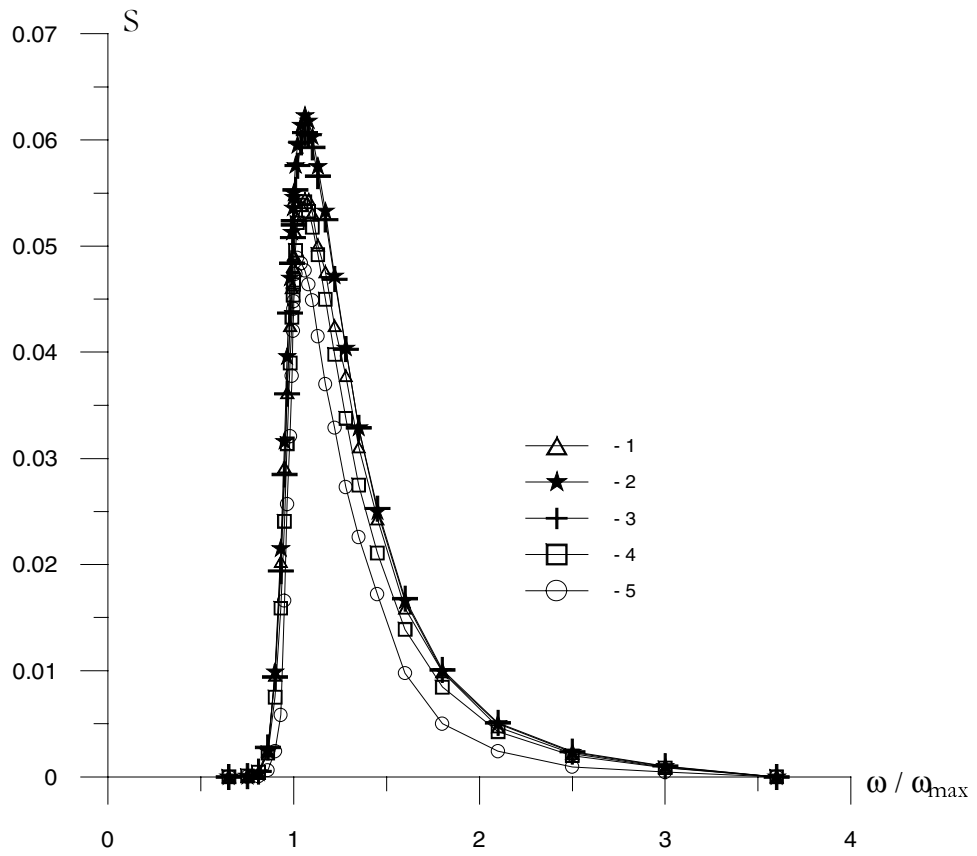

Fig. 3. Numerical value of frequency spectrum at $t=30 \mathrm{~h}, 1$-solution without non-linear energy transfer action; 2 - the second order method, time step $\Delta t=3$ min; 3- spreading method $\Delta t=$ $1 \mathrm{~h} ; 4-\Delta t=3 \mathrm{~h} ; 5-\Delta t=6 \mathrm{~h}$.

\section{Conclusions}

The optimal numerical realization of the energy balance equation in wind wave models is proposed. The scheme is separated into two parts: the energy propagation numerical realization and the numerical source term integration. The energy propagation numerical realization is based on the semi-Lagrangian numerical method. The original correction term is derived to remove the garden-sprinkler effect of the solution of energy balance equation for the case of wave propagation on spherical 
surface. The method results proved to be much closer to the analytical solution than the finite difference schemes. The method is absolute stabile. Its higher accuracy is achieved by using larger propagation time steps (three hours and more). The main feature of the method is that it may be applicable to solve the energy balance equation with a fine space grid by using larger time step than it is allowed by the traditional finite-difference schemes.

The second part of the total scheme is based on a spreading numerical method. It uses the semi-analytical solution for integration source term, which includes the wind wave input, dissipation term, and exact non-linear energy transfer function. The method gives reliable and stable results for time steps up to three hours and more. The new technique yields

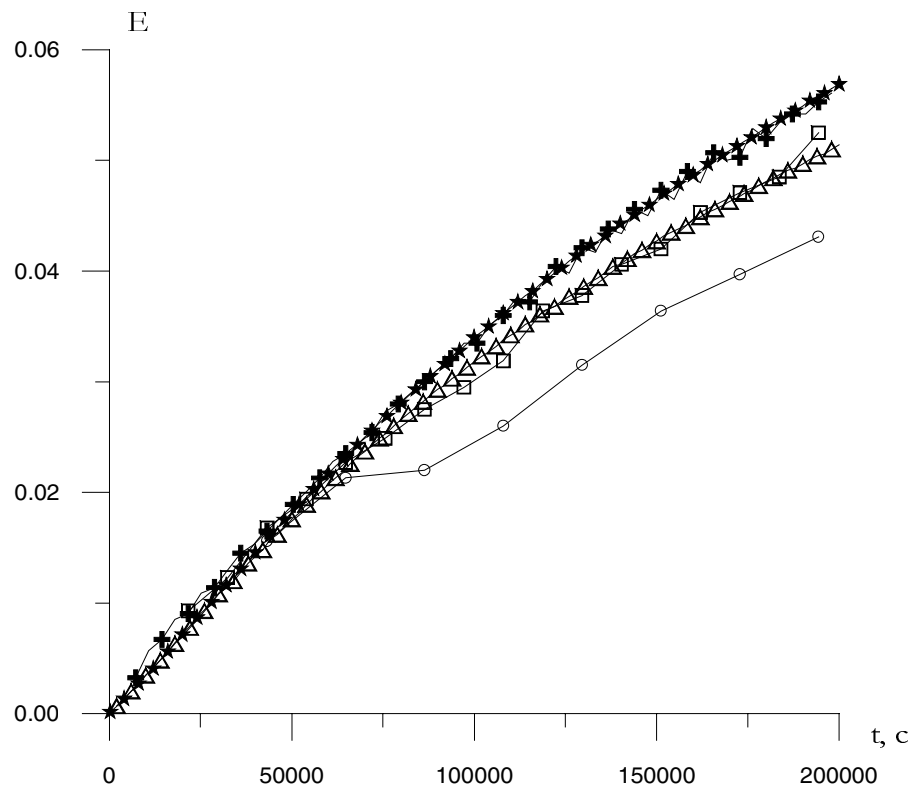

Fig. 4. Total energy evolution in time (designations are the same as to Fig.3)

accurate results and has the additional advantage of being numerically stable, thereby allowing to use a large time step (three hours and more).

The method can be regarded as a general alternative for the numerical realization of energy balance equation in the models. It gives opportunity to approach the time step of numerical integration of the energy balance equation to the time step of the wind speed information input in the numerical wind wave simulation to solve operational problem in the optimal way.

Acknowledgements. The study is supported by the Grants: RFBR 01-05-64846, INTAS-99-666, INTAS-01-025, INTAS-01-234, INTAS-01-2156. 


\section{References}

1. Booij, N. and Holthuijsen, L.H.: Propagation of Ocean Waves in Discrete spectral wave model. Journal of Comput. Phys. 68, (1987), 307-326

2. Burgers, G.: A guide to the Nedwam wave model. Scient.Rep, WR-90-04, KNMI, De Bilt.

3. Komen G.J., Cavaleri, L., Donelan, M., Hasselmann, K., Hasselman, S., Janssen, P.A.E.M Dynamics and modeling of ocean waves, Cambridge University press, (1994), 532

4. Lavrenov, I.V.: Wind Waves in Ocean. Dynamics and numerical simulation. Springer, (2003), 375

5. Lavrenov, I.V., Onvlee, J.: New approach for reduction of the garden-sprinkler effect in third generation wave models, Scient.Rep. KNMI, De Bilt. (1993)

6. Tolman, H.L.: Effects of numeric on the physics in a third-generation wind-wave model, J.Phys.Ocean.22, (1992), 1095-1111

7. Zambresky, L.F.: A verification study of global WAM model December 1987 - November 1988, ECMWF Technical Report 63, ECMWF, Reading, (1989), 86 